\title{
Limited Delay Preemption Based Priority in Differentiated Optical Burst Switching Networks with Small Buffers
}

\author{
Jiuru Yang, Hong'an Ye \\ Key Lab of Electronics Engineering, College of Heilongjiang Province, Heilongjiang University, Harbin, China \\ Email: yangjr@hlju.edu.cn
}

Received June 2013

\begin{abstract}
The composite scheme based on preemption and small buffers is an efficient method for contention resolution. To support services differentiation, it is the first time that the analytical model of delay preemption based priority is built. Further, in order to guarantee the low-loss requirement for high priority bursts, an improved scheme is proposed and investigated by limiting the buffered right of low priority bursts within the specific traffic states. The simulation results show that, without the deterioration of blocking performance, there is more than $40 \%$ reduction on burst loss being achieved under the condition $\rho=1.0$ for high priority bursts.
\end{abstract}

Keywords: Optical Burst Switching; Preemption; Small Buffers; Blocking

\section{Introduction}

Since the concept of optical burst switching (OBS) is proposed by Qiao in 1999, it has been regarded as one of promising solutions for future optical Internet [1]. In OBS, due to one-way reservation behavior, the contention among bursts is inevitable, and much works have been dedicated to contention resolution by means of tunable wavelength converters (TWCs) and optical buffers, i.e., fiber delay lines (FDLs) [2-4]. However, it is determinate that the abundant usage of TWCs and FDLs at core nodes will bring the problems of not only the huge complexity in configuration, but also the large energy consumption because of the astonishing traffic increase recently [5].

Under the wave of green networks, some practical and applicable schemes based small buffers (the length is about tens of packets) are concerned. In [6,7], the cost-efficiency based feedback buffer configuration is studied. Centering real-time and TCP traffic, the effects of size of small buffers on data loss are analyzed by Rouskas [8]. And to avoid burst-discarding, preemption is introduced through pre-reserving or post-reserving channel for the collided and buffered bursts [9,10]. Alternatively, [11] mitigates the performance degradation resulting from small buffers by smoothing traffic burstiness at edge nodes. Also, combine pre-reservation and segmentation, the authors proposed delay preemption to further reduce the packets loss probability of OBS [12]. In this paper, to support services differentiation, it is the first time that the analytical model on delay preemption based priority (DPP) is built. Moreover, on the basis of DPP, an improved scheme called Limited Delay Preemption Based Priority (LDPP) is proposed to guarantee the quantity of service (QoS) requirements of both mission-critical and delaysensitive services by constraining the right of low priority bursts to enter FDLs within the specific traffic states.

\section{Analytical Model of Delay Preemption Based Priority}

Assuming the mean arrival rate and service time of bursts are $\lambda$ and $1 / \mu$, respectively. And the incoming packets with the common destinations are aggregated into a burst at edge nodes. For simplicity, the aggregated threshold in length $L_{b}$ for all bursts is the same and fixed. In delay preemption with single-class, when more than two bursts transmit and switch in the same wavelength channel simultaneously, a channel contention occurs. Given the FDLs is idle, the contended burst can be buffered and preempt the channel in advance [12].

We then define $\rho=\lambda / W \mu$ is offered load and $P_{D}$ denotes the total burst loss probability, where $W$ is the number of wavelength in each fiber link. Thus, the probability of channel usage at core nodes can be represented as

$$
u=\rho\left(1-P_{D}\right)
$$

Likewise, in two-class system, we define both mission-critical and delay-sensitive services with high priority, denoted by $B_{h i g}$, and other bursts are with low priority, 
denoted by $B_{\text {low }}$. Their offered loads are $\rho_{1}$ and $\rho_{2}$ respectively, and $\rho=\rho_{1}+\rho_{2}$. Moreover, the probabilities of channel usage of $B_{\text {hig }}$ and $B_{\text {low }}$ are denoted by $u_{1}$ and $u_{2}$, and $u=u_{1}+u_{2}$. Further, define $\lambda_{1}=c_{1} \cdot \lambda$ and $\lambda_{2}=c_{2} \cdot \lambda$, where $c_{1}=\rho_{1} / \rho$ and $c_{2}=\rho_{2} / \rho$ are the load ratios of $B_{\text {hig }}$ and $B_{\text {low }}$ in networks. Then, owing to $c_{1}+c_{2}=1$, the probabilities of channel usage in two-class system are written as

$$
u_{1}=c_{1} u=c_{1} \rho\left(1-P_{D}\right)
$$

and

$$
u_{2}=c_{2} u=c_{2} \rho\left(1-P_{D}\right)
$$

Next, the basics of delay preemption based priority (DPP) are shown in Figure 1, where $b$ is the FDLs length and $r$ is blocking length. Apparently, $b$ should be larger than $r$ to avoid burst congestion in buffer. According to [13], the expectation of $r$ can be presented as

$$
E[r]=\frac{\overline{L_{b}}}{2}+\frac{\delta_{L_{b}}^{2}}{2 L_{b}}
$$

where $\overline{L_{b}}$ is the mean of $L_{b}$, and $\delta_{L_{b}}^{2}$ is its variance. Because $L_{b}$ is fixed in this paper, namely $\overline{L_{b}}=L_{b}$, it means $\delta_{L_{b}}^{2} \rightarrow 0$, and $E[r] \approx L_{b} / 2$. Besides, based the result in [8], we design $b=L_{b}$. Thus, $E[r]=b / 2$.

Furthermore, in Figure 1(a), we see a channel contention occurs between the high priority burst $B_{h i g, i}$ and low priority burst $B_{\text {low }, j}$. Under FIFO rule (first in first out), $B_{\text {low }, j}$ cuts through the channel directly. In this case, if the FDL is in the idle/free state, the blocked burst $B_{h i g, i}$ can be buffered and preempt the channel $b-r+L_{b}$ for itself in advance. Otherwise, $B_{h i g, i}$ would be simply dropped and retransmitted. Hence, for high priority, its loss probability $P_{D, 1}$ can be written as

(a)
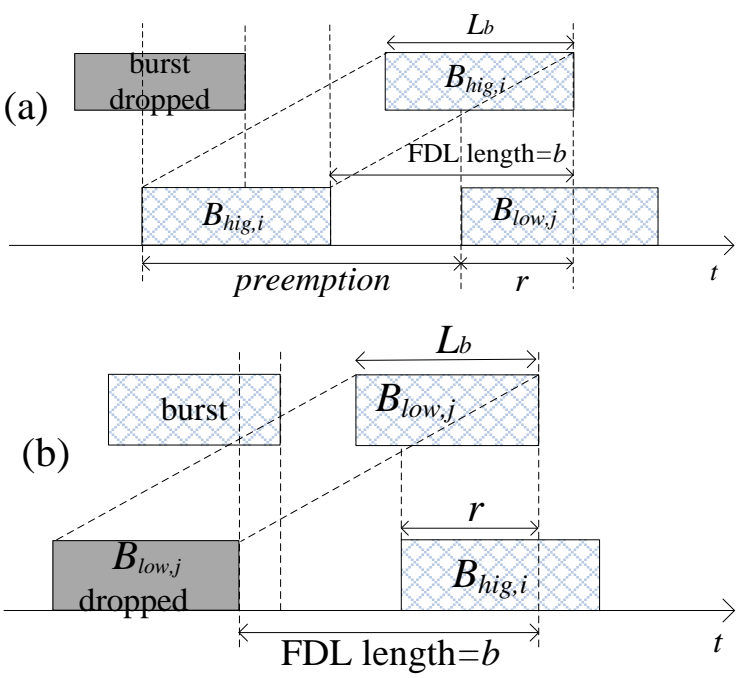

Figure 1. Principles of delay preemption based priority, (a) blocking of high priority burst; (b) blocking of low priority bursts.

$$
\begin{aligned}
P_{D, 1} & =c_{1} \text { Pr. }\{\text { channel busy } \bigcap \text { buffer busy }\} \\
& =c_{1} \cdot u P_{F D L}
\end{aligned}
$$

where $P_{F D L}=P_{1, F D L}+P_{2, F D L}$ represents the probability of buffer usage, $P_{1, F D L}$ and $P_{2, F D L}$ are the probabilities of buffer usage from $B_{\text {hig }}$ and $B_{\text {low }}$ respectively. Based on [14], the idle probability within the inter-arrival time $t$ can be represented as $e^{-\lambda t}$. Then, $P_{1, F D L}=1-e^{-\lambda_{1} b}$ and $P_{2, F D L}=1-e^{-\lambda_{2} b}$. In other hand, see Figure 1(b), only the right to be buffered is given to low priority. That means, though it has been delayed $b$ in FDLs, $B_{\text {low, } j}$ would be discarded if a burst arrived within the void $b-r$. In addition, because of no priority to enter the buffer, an extra burst loss for $B_{\text {low }}$ can be brought when the buffer is idle but pre-reserved by high priority bursts. Therefore, the burst loss probability of low priority can be calculated as

$$
\begin{aligned}
P_{D, 2}= & c_{2} \operatorname{Pr} .\{\text { channel busy } \bigcap \text { buffer busy }\} \\
& +\operatorname{Pr} .\{\text { channel busy } \bigcap \text { buffer free }\} \cdot c_{1} \\
= & c_{2} \cdot u P_{F D L}+u\left(1-P_{F D L}\right) \cdot c_{1} \\
= & u \cdot\left[c_{1}+\left(c_{2}-c_{1}\right)\left(P_{1, F D L}+P_{2, F D L}\right)\right]
\end{aligned}
$$

where $\left(1-P_{F D L}\right)$ is the free probability of buffer. Because $P_{D}=P_{D, 1}+P_{D, 2}$ and $c_{1}+c_{2}=1$, the total loss probability in DPP will be

$$
\begin{aligned}
P_{D} & =c_{1} \cdot u P_{F D L}+u \cdot\left[c_{1}+\left(c_{2}-c_{1}\right)\left(P_{1, F D L}+P_{1, F D L}\right)\right] \\
& =u \cdot\left[c_{1}+c_{2}\left(P_{1, F D L}+P_{2, F D L}\right)\right]
\end{aligned}
$$

Combine Equations (1) and (4), we get

$$
P_{D}=\frac{\rho\left[c_{2}\left(P_{1, F D L}+P_{2, F D L}\right)+c_{1}\right]}{\rho\left[c_{2}\left(P_{1, F D L}+P_{2, F D L}\right)+c_{1}\right]+1}
$$

Then, with the various load ratios, the blocking performance of DPP is investigated from a typical simulation scenario used in [12], and $b$ is designed as $80 \mu \mathrm{s}$ specially. In Figure 2, there are two apparent natures on data loss in DPP. First, compared to the result of single-class, it is certain that there are the obvious reductions on loss probability existing in all traffic states, especially when $c_{1}$ is a smaller value. Secondly, we find the value of $P_{D}$ is positive proportional to the variant $c_{1}$. For instance, when $c_{1}=0.2$ or 0.3 , a very low $P_{D}$, less than $10^{-3}$, is obtained in light load $(\rho<0.3)$. Instead, for $c_{1}=$ 0.5 , the burst loss probability is increased quickly, and reaches $2 \times 10^{-1}$ at $\rho=0.7$, which is almost equal to the result of single-class. Although as the minority of networks, the load ratio of high priority is general less than $30 \%$ [15], we should strongly notice that, being in heavy load, a higher $P_{D}$ is also demonstrated when $c_{1}$ is a small value (e.g. when $c_{1}=0.2, P_{D}$ is equal to $10^{-2}$ at $\rho=0.7$ ). In one word, DPP is suboptimal due to the fact a higher loss probability still exists in high traffic states. 


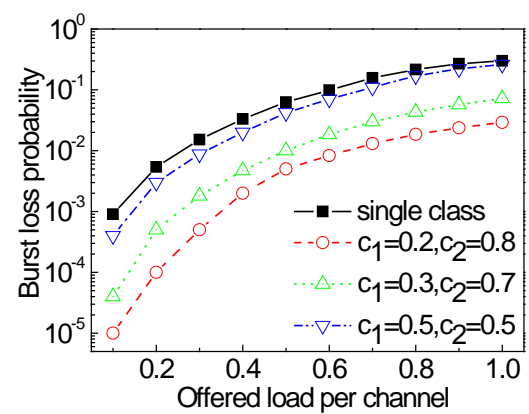

Figure 2. Total loss of DPP.

Based on Equations (2)-(4), we take a further analysis for high and low priority on blocking performance, and a typical value 0.3 is selected for $c_{1}$. The numerical results in Figure 3 indicate that $B_{\text {low }}$ is the main contributor of the total burst loss probability. And for high priority bursts, two apparent natures on $P_{D, 1}$ are exhibited in different traffic states. In light load, its loss probability is just about $1 / 10$ of $P_{D, 2}$. But, with the increase of offered load, $P_{D, 1}$ is soared dramatically and reaches $0.33 P_{D}$ under the condition $\rho=1.0$. From Equation (2), the loss of high priority is mainly dependant on the parameters $u$ and $P_{F D L}$. Considering the majority nature, we infer that such higher $P_{D, 1}$ in heavy load is resulted from the over-occupancy of the huge low priority bursts on channel and buffer.

\section{Limited Delay Preemption Based Priority}

According to the above analysis, the proposed DPP scheme cannot guarantee the low-loss requirements of high priority bursts because the buffer is unfairly over-occupied by low priority. To overcome the weakness of DPP, it is necessary to take a limitation on buffer usage of low priority within high traffic states. Therewith, an improved scheme called Limited Delay Preemption Based Priority (LDPP) is proposed. In LDPP, the buffered right for low priority bursts is deprived when offered load reaches the designed threshold $\rho_{t h}$. Thereby, given the channel contention takes place, the burst with low priority will be discarded, whether the buffer is in the idle state or not. We then get $P_{D, 2}=c_{2} u$ when $\rho>\rho_{t h}$. In addition, owing to $P_{2, F D L}=0$, the loss probability of high priority bursts $P_{D, 1}$ is reduced as

$$
P_{D, 1}=c_{1} u \cdot P_{1, F D L} \rho>\rho_{t h}
$$

Therefore, in LDPP, the total loss will be improved and Equation (5) is substituted by

$$
P_{D}= \begin{cases}\frac{\rho\left[c_{2}\left(P_{1, F D L}+P_{2, F D L}\right)+c_{1}\right]}{1+\rho\left[c_{2}\left(P_{1, F D L}+P_{2, F D L}\right)+c_{1}\right]} & \rho \leq \rho_{t h} \\ \frac{\rho\left(c_{1} P_{1, F D L}+c_{2}\right)}{1+\rho\left(c_{1} P_{1, F D L}+c_{2}\right)} & \rho>\rho_{t h}\end{cases}
$$

From Equation (7), the performance evaluation of LDPP on blocking is developed and the simulation results are shown in Figures $\mathbf{4}$ and 5. Compared to DPP, in the range of $\rho_{t h}=0.3-0.7$, LDPP obtained a blocking improvement, at least $20 \%$, under the condition $\rho=1.0$. Besides, we find that, the lower threshold $\rho_{t h}$ is selected, the smaller loss probability is gained (see Figure 4). Whereas, considering the loss requirements of $B_{\text {low }}$ and the buffer usage efficiency in light load, the value of load threshold should not be too small.

Here, an eclectic value $\rho_{t h}=0.5$ is chosen, and the blocking performance of high and low priority is again investigated. Comparatively, on one hand, the loss probability of $B_{\text {low }}$ in LDPP is not deteriorated in all traffic states though the buffered right has been deprived partially. On the other hand, the blocking performance of high priority bursts is improved, and more than $40 \%$ reduction on $P_{D, 1}$ under the condition $\rho=1.0$ is shown in Figure 5. In consequence, the total loss probability $P_{D}$ is

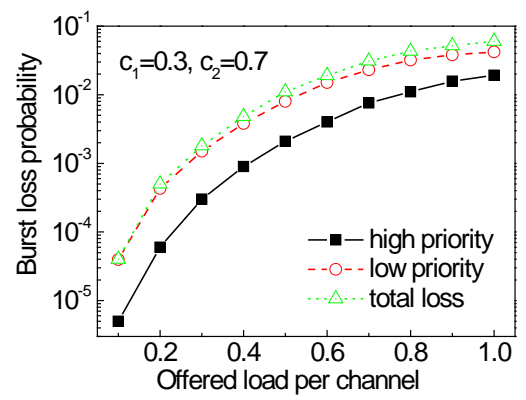

Figure 3. Blocking of high priority and low priority in DPP.

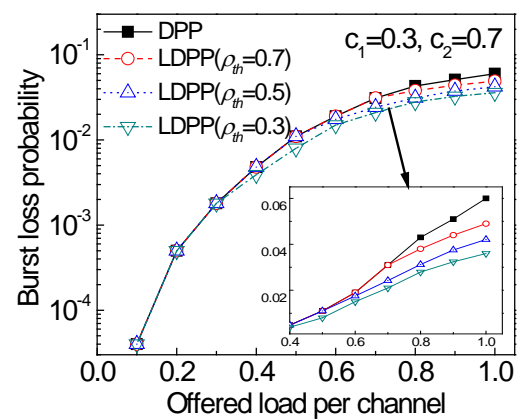

Figure 4. Total loss of LDPP.

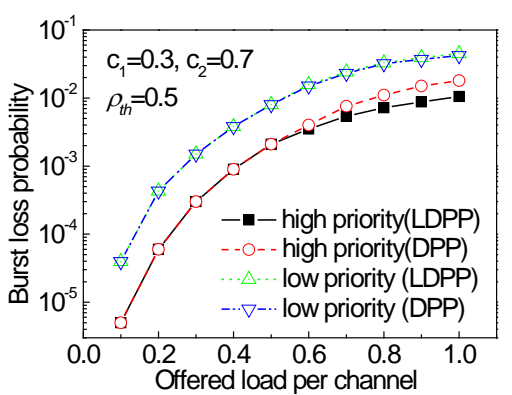

Figure 5. Blocking comparison between DPP and LDPP. 
also reduced about $20 \%$ in heavy load, which has been proved in Figure 4.

\section{Summary}

In this paper, to support services differentiation, the analytical model of delay preemption based priority is developed, and an improved scheme LDPP is proposed to alleviate the over-occupancy of buffer from low priority in heavy load. The simulation results show that, through taking a tradeoff on the buffer usage of bursts, more than $40 \%$ reduction of high priority on blocking is obtained in LDPP, without the deterioration of total blocking. And our future work will focus on the evaluation of LDPP in terms of fairness and energy consumption.

\section{Acknowledgements}

This work is supported by the Heilongjiang Province Natural Science Foundation (No. 201009).

\section{REFERENCES}

[1] P. P. Marino and F. Neri, "On the Myths of Optical Burst Switching," IEEE Transactions on Communications, Vol. 59, No. 9, 2011, pp. 2574-2584. http://dx.doi.org/10.1109/TCOMM.2011.063011.100192

[2] C. Wu and S. Xiao, "Improved Optical Packet Switching Structure with Recirculation Buffer and Feedback Tunable Wavelength Converter," Chinese Optics Letters, Vol. 7, No. 5, 2009, pp. 384-386.

http://dx.doi.org/10.3788/COL20090705.0384

[3] G. Marchetto, "High-Priority First Transmission to Efficiently Support Service Differentiation in Just-in-Time OBS Networks," Journal of Optical Communications and Networking, Vol. 2, No. 12, 2010, pp. 1031-1041. http://dx.doi.org/10.1364/JOCN.2.001031

[4] L. Hou, Y. Lu, J. Wang, Y. Ji and Y. Hua, "Extending Path Computation Element for Lightpath Restoration in Wavelength-Switched Optical Networks," Chinese Optics Letters, Vol. 8, No.2, 2010, pp. 142-145. http://dx.doi.org/10.3788/COL20100802.0142

[5] R. S. Tucker, "Green Optical Communication-Part II: Energy Limitations in Networks," IEEE Journal of Selected Topics in Quantum Electronics, Vol. 17, No. 2,
2011, pp. 261-274. http://dx.doi.org/10.1109/JSTQE.2010.2051217

[6] J. Choi and M. Kang, "Service Differentiation Using Hybrid Shared Optical Buffers in Transparent Optical Networks," Optics Express, Vol. 14, No. 12, 2006, pp. 5079-5091. http://dx.doi.org/10.1364/OE.14.005079

[7] C. McArdle, D. Rafani, T. Curran, A. Holohan and L.P. Barry, "Renewal Model of a Buffered Optical Burst Switch,” IEEE Communications Letters, Vol. 15, No. 1, 2011, pp. 91-93. http://dx.doi.org/10.1109/LCOMM.2010.120610.101196

[8] A. Vishwanath, V. Sivaraman and G. N. Rouskas, “Anomalous Loss Performance for Mixed Real-Time and TCP Traffic in Routers with Very Small Buffers,” IEEE/ACM Transactions on Networking, Vol. 19, No. 4, 2011, pp. 933-946. http://dx.doi.org/10.1109/TNET.2010.2091721

[9] A. Rostami and S. S. Chakraborty, "On Performance of Optical Buffers with Specific Number of Circulations," IEEE Photonics Technology Letters, Vol. 17, No. 7, 2005, pp. 1570-1572. http://dx.doi.org/10.1109/LPT.2005.848548

[10] N. Akar and K. Shoraby, "Retrial Queuing Models of Multi-Wavelength FDL Feedback Optical Buffers,” IEEE Transactions on Communications, Vol. 59, No. 10, 2011, pp. 2832-2840. http://dx.doi.org/10.1109/TCOMM.2011.071111.100521

[11] V. Sivaraman, H. Elgindy, D. Moreland and D. Qstry, "Packet Pacing in Small Buffer Optical Packet Switched Networks," IEEE/ACM Transactions on Networking, Vol. 17, No. 4, 2009, pp. 1066-1079. http://dx.doi.org/10.1109/TNET.2008.2005622

[12] J. Yang, "Burst Contention Resolution Strategy based Delay Preemption,” Acta Optica Sinica, Vol. 28s, No. 12, 2008, pp. 209-212.

[13] A. Detti, V. Eramo and M. Listanti, "Performance Evaluation of a New Technique for IP Support in a WDM Optical Network: Optical Composite Burst Switching (OCBS)," Journal of Lightwave Technology, Vol. 20, No. 2, 2002, pp. 154-165. http://dx.doi.org/10.1109/50.983228

[14] K. Leonard, "Queueing System I: Queueing Theory," John Wiley, New York, 1975.

[15] C. Politi and A. Stavads, "Routing in Meshed and Clustered Optical Networks," Proceedings of ECOC, Torino, 2010, p. 5.06 